\title{
Stability and neutralization capacity of a novel mosaic HIV-1 gp140 trimer in a guinea pig model
}

\author{
JP Nkolola ${ }^{\text {* }}$, JM Kovacs ${ }^{2}$, B Korber ${ }^{3}$, B Chen ${ }^{2}$, M Seaman $^{4}$, D Barouch ${ }^{1}$ \\ From AIDS Vaccine 2012 \\ Boston, MA, USA. 9-12 September 2012
}

\section{Background}

The generation of globally relevant HIV-1 immunogens mimicking native, trimeric Envelope (Env) structure, remains a major challenge for HIV-1 vaccine development. We identified a mosaic Env sequence, originally designed to optimize cellular immunologic coverage of global HIV-1 sequence diversity, which was capable of forming biochemically stable trimers. We assessed this mosaic Env gp140 trimer in guinea pig immunogenicity studies compared to our previously reported biochemically stable C97ZA012 (clade C) trimer.

\section{Methods}

Stable Env gp140 trimer derived from a synthetic mosaic sequence was stabilized with the T4-fibritin C-terminal trimerization tag and produced in $293 \mathrm{~T}$ cells via PEI transfection. Characterization was performed by Western blotting, size-exclusion chromatography and surface plasmon resonance (SPR). Guinea pigs were immunized three times with $100 \mu \mathrm{g}$ of mosaic or clade C gp140 protein trimer in $\mathrm{CpG} /$ Emulsigen adjuvants. Antibody responses were determined by ELISA and TZM.bl neutralizing antibody assays.

\section{Results}

Stabilized mosaic gp140 trimer exhibited a single band by Western blotting, single peak by size-exclusion chromatography both after production, a freeze-thaw cycle and 7day incubation at $4^{\circ} \mathrm{C}$. SPR analyses revealed mosaic gp140 binding with bNAb VRC01. The trimeric mosaic gp140 immunogen elicited high-titer antibodies in guinea pigs by ELISA and high-titer, cross-clade neutralization activity against tier 1 viruses. When compared to clade $\mathrm{C}$ gp140 trimer, NAb responses generated by mosaic gp140 trimer were 8.2- and 3.7-fold higher against clade
$B$ viruses (SF162.LS and Bal.26, respectively) but were 10.3- and 46.7-fold lower against clade $A$ and $C$ viruses (DJ263.8 and MW965.26, respectively).

\section{Conclusion}

A novel, foldon-stabilized mosaic gp140 trimer elicits high-titer binding antibodies as well as high-titer, crossclade neutralization of tier 1 viruses. The profile of the NAbs elicited by the mosaic trimer differed from that elicited by the clade $\mathrm{C}$ trimer. Further exploration and refinement of this concept may contribute to the development of a globally relevant Env immunogen.

\section{Author details}

'BIDMC / Ragon Institute of MGH, MIT and Harvard, Boston, MA, USA. ${ }^{2}$ Children's Hospital Boston, Boston, MA, USA. ${ }^{3}$ Los Alamos National Laboratory, Los Alamos, NM, USA. ${ }^{4}$ Beth Israel Deaconess Medical Center, Boston, MA, USA.

Published: 13 September 2012

doi:10.1186/1742-4690-9-S2-P299

Cite this article as: Nkolola et al:: Stability and neutralization capacity of a novel mosaic HIV-1 gp140 trimer in a guinea pig model. Retrovirology 2012 9(Suppl 2):P299. 